\title{
Intervenções de enfermagem para alta de paciente prostatectomizado: uma revisão bibliográfica
}

\author{
Nursing interventions for discharge of prostatectomized patient \\ Intervenciones de enfermería para el alta del paciente prostatectomizado
}

Graziele Moreira Silva ${ }^{1 *}$, Tayane Raquel Muniz de Campos ${ }^{1}$, Andreia Andrade dos Santos ${ }^{1}$, Márcio Antônio Resende 1 .

\section{RESUMO}

Objetivo: O presente estudo buscou demonstrar a importância da utilização do Processo de Sistematização de Enfermagem no cuidado ao paciente prostatectomizado. Revisão bibliográfica: No cuidado aos pacientes prostatectomizados, a enfermagem tem um papel importante no preparo para a alta hospitalar, uma vez que os pacientes deixam o hospital com muitas dúvidas sobre o funcionamento do aparelho urinário e reprodutor além de apresentarem carência emocional e psicológica. O Processo de Enfermagem (PE) fundamenta-se como uma formação conceitual sólida que proporciona o prosseguimento do cuidado e a qualidade da assistência de enfermagem, sendo assim, é preciso que a equipe atenda às necessidades desse paciente de forma a tranquilizá-lo para ir embora em plenas condições de finalizar seus cuidados. Considerações finais: Ficou claro o peso positivo que boa orientação, comunicação e cuidado têm na prática de Enfermagem e espera-se que esse estudo contribua para a construção acadêmica de profissionais de Enfermagem que se dediquem a esse cuidado tão necessário.

Palavras-Chave: Câncer de próstata, Processo de sistematização de enfermagem, Cuidados pós-operatórios, Alta hospitalar.

\begin{abstract}
Objective: This study aimed to demonstrate the importance of using the Nursing Systematization Process in the care of prostatectomized patients. Literature review: In the care of prostatectomized patients, nursing plays an important role in preparing for hospital discharge, since patients leave the hospital with many doubts about the functioning of the urinary and reproductive tracts, besides presenting emotion and psychological deficiency. The nursing process is founded as a solid conceptual formation that proportions the care process and the quality of nursing, so it is necessary that the team must meet the needs of this patient in order to reassure him to leave in full condition to finalize his care. Final considerations: It is clear the positive weight that good guidance, communication and care have in nursing practice and it is expected that this study contributes to the academic construction of nursing professionals who are dedicated to this much needed care.
\end{abstract}

Key words: Prostate cancer, Nursing care systematization, Postoperative care, Hospital discharge.

\section{RESUMEN}

Objetivo: Este estudio quiere demostrar la importancia de la utilización del Proceso de Sistematización de Enfermería al cuidado de pacientes prostatectomizado. Revisión bibliográfica: En el cuidado a los pacientes prostatectomizados, la enfermería tiene un importante papel al preparar para el alta hospitalario, una vez que los pacientes dejan el sanatoriocon muchas dudas sobre el funcionamiento del aparato urinario y reproductor,

${ }^{1}$ Centro Universitário Presidente Tancredo de Almeida Neves - (UNIPTAN), São João del-Rei - MG.

*E-mail: grazielegms@yahoo.com.br 
además de presentar carencia emocional y sicológica. El proceso de enfermería (PE) se fundamenta como una sólida formación conceptual que proporciona el proseguimiento del cuidado y lá calidad de lá asistencia de enfermería, así siendo, es necesario que el equipo atienda a las necesidades de ese paciente de maneira a tranquilizarlo para que se vaya en plenas condiciones de finalizar sus cuidados. Consideraciones finales: Quedó muy claro el peso positivo que buena orientación, comunicación y cuidado tienen en la práctica de Enfermería y se espera que este estudio contribuya para la construcción académica de profesionales de Enfermería que se dediquen a ese tan necesario cuidado.

Palabras clave: Cáncer de próstata, Sistematización del cuidado de enfermería, Cuidados postoperatorios, Alta hospitalaria.

\section{INTRODUÇÃO}

Entre as doenças relacionadas à saúde do homem, atualmente, prevalecem aquelas que envolvem a próstata, destacando a hiperplasia benigna (HPB) e maligna (câncer). A baixa procura da população masculina na Atenção Primária - que tem como objetivo a Promoção da Saúde e a diminuição de agravos faz com que essas patologias sejam diagnosticadas em estágios avançados, e o tratamento para ambas as doenças dependerá do quadro clínico do paciente. As opções vão desde a conduta conservadora, como a observação e o tratamento medicamentoso, até o procedimento cirúrgico, denominado prostatectomia (SMELTZER SC e BARE BG, 2011).

No cuidado ao paciente prostatectomizado, a enfermagem tem papel importante no preparo para alta hospitalar, uma vez que os pacientes deixam o hospital com dúvidas sobre o funcionamento do aparelho urinário e reprodutor, além de apresentarem carência emocional e psicológica (MATA LRF e NAPOLEÃO AL, 2010). Sendo assim, é preciso que a equipe atenda às necessidades do paciente de forma a deixá-lo tranquilo para ir embora em plenas condições de finalizar seus cuidados, contando, preferencialmente, com a ajuda de enfermeiros capacitados em sanar suas questões e psicólogos aptos a ajudar nas demandas emocionais.

O enfermeiro deve preocupar-se com ações planejadas para assegurar ao paciente e à sua família cuidado amplo, considerando aspectos físicos, psicológicos, sociais, culturais e espirituais. Nesse cenário, o Processo de Enfermagem (PE) fundamenta-se como uma formação conceitual sólida que proporciona 0 prosseguimento do cuidado e a qualidade da assistência de enfermagem (ALMEIDA MDA, et al.,2012).

Essa problemática, que envolve as dúvidas e expectativas dos pacientes prostatectomizados, correlacionada ao conhecimento da importância da utilização do PE como um método de instrumentalização de cuidados durante a alta hospitalar, despertou nas autoras o interesse em desenvolver este estudo, com o objetivo de retratar a importância do PE ao paciente submetido à prostatectomia na alta hospitalar.

Este estudo tem por finalidade revisar na literatura a importância das intervenções de enfermagem, através da implementação do PE, aos pacientes submetidos a cirurgia de prostatectomia radical para alta hospitalar.

\section{REVISÃO BIBLIOGRÁFICA}

\section{Câncer de próstata}

O câncer (CA) de próstata é o tumor que afeta a próstata, e é considerado a neoplasia mais comum em homens, seguido do CA de pele não-melanoma e a segunda causa principal de morte por câncer em homens no Brasil. As células são partes minúsculas do corpo humano, e se multiplicam rapidamente ao longo da vida, ocorrendo a substituição das mais antigas pelas novas e assim sucessivamente. Em alguns casos, ocorre um crescimento anormal e desenfreado das células, sendo capazes de invadir outros tecidos formando os tumores, podendo ser benignos, que são os não cancerígenos, ou malignos, denominados câncer. (MS, 2018).

A próstata é a maior glândula acessória do sistema genital masculino e tem, aproximadamente, as dimensões e a forma de uma castanha, ficando situada abaixo da bexiga e envolvendo a parte inicial da uretra. A face posterior da próstata está em contato com o reto, o que permite que se perceba a consistência e o 
volume da glândula por meio do toque retal; ainda, anteriormente à próstata e inferiormente à bexiga, encontram-se, de cada lado, a vesícula seminal e o canal deferente, que atravessam o órgão retentor de urina para desembocar na uretra e têm como função a produção de parte do sêmen eliminado pelo homem (MOORE KL e DALLEY AF, 2001).

Segundo o Instituto Nacional do Câncer (2019), o CA de próstata é considerado o câncer da terceira idade, cerca de $75 \%$ dos casos no mundo ocorrem a partir dos 65 anos. O aumento na taxa de incidência do Brasil pode ser relativamente justificado pelo avanço dos métodos diagnósticos, pelo desenvolvimento dos sistemas de informação, e pelo aumento na expectativa de vida. (INCA, 2019).

Apesar de não se saber ao certo a causa do câncer de próstata, a idade é um dos marcadores de risco de maior relevância, uma vez que a doença acomete principalmente a população masculina acima de 50 anos. Histórico familiar de primeiro grau (pais, filhos e irmãos) e peso corporal elevado são outros marcadores de importância (MS, 2018). As evidências em se adotar práticas saudáveis, como, comer uma variedade de frutas, verduras e vegetais, praticar atividade física, manter o peso corporal adequado e evitar o consumo de cigarro e bebidas alcoólicas são consideradas métodos de prevenção, para diminuir o risco de câncer de próstata. (INCA, 2019).

Conforme preconizado pelo (MS, 2018), a partir dos 50 anos todos os homens devem realizar o exame de dosagem de antígeno prostático específico (PSA), que avalia a quantidade de antígeno prostático específico, e o toque retal.

O exame de PSA, é importante para avaliar se há doença tumoral ativa na próstata e para monitorar tumores já conhecidos (AMERICAN CANCER SOCIETY, 2019). Já o toque retal é imprescindível para averiguar alterações de forma e consistência da próstata na busca ativa de problemas na região, de modo a detectar qualquer sinal precocemente e empreender melhor plano preventivo. Ademais, se houver histórico de CA na família, é recomendada a realização dos exames a partir dos 45 anos.

No estágio inicial da doença, os sinais e os sintomas do câncer aparecem de forma silenciosa, aumentando a importância de se realizar periodicamente os exames de rastreamento. Na fase mais avançada, já ocorre manifestação intensa do quadro, em que é possível citar o aparecimento de sinais e sintomas, tais como: disúria (dor ao urinar), polaciúria (aumento patológico do número de micções), noctúria (necessidade de se levantar durante a noite para urinar), hematúria (presença de sangue na urina) e dor óssea. Esses problemas podem provocar complicações, como infecção generalizada ou insuficiência renal. (INCA, 2019). O Sistema Único de Saúde (SUS) oferece de forma gratuita e integral, além dos exames de diagnostico, o tratamento independente de sua modalidade (MS, 2018).

Os tratamentos para o câncer de próstata variam de acordo com o estágio da doença. Os estágios vão do I ao IV, assim subdivididos: no estágio I, os tumores são pequenos e não ultrapassam a próstata, sendo assintomáticos; o estágio II apresenta tumores ainda na glândula, porém maiores e com elevação do PSA; no estágio III, os tumores ultrapassam a próstata, mas não atingem a bexiga ou linfonodos e possuem maior chance de recidiva; já no estágio IV, os tumores se disseminam para bexiga e reto e, geralmente, podem ser apenas tratados, mas não curados (AMERICAN CANCER SOCIETY, 2019).

Quando a doença se encontra localizada, isto é, quanto atinge somente a próstata, sem progredir para outros órgãos, o tratamento indicado consiste em cirurgia, radioterapia e observação vigilante. Quando a doença alcança outros órgãos, a cirurgia ou a radioterapia, em combinação com terapia hormonal, passa a ser o tratamento mais apropriado (MS, 2018).

A escolha do tratamento deve ser feita por meio de diálogo entre médico e paciente, levando em consideração o estágio da doença e a idade de cada indivíduo afetado. Para mais, vale ressaltar a importância de informar os riscos e os benefícios de cada tratamento para se chegar em decisão concreta (MATA LRF e NAPOLEÃO AL, 2010).

Sabe-se que, quanto mais precoce for a detecção do câncer, maiores as chances de cura da doença. Porém, a baixa adesão da população masculina aos serviços de saúde faz com que o diagnóstico seja constatado em estágio já avançado da doença. Pinheiro RJ, et al., (2002) apontam que 62,3\% das mulheres brasileiras pesquisadas pelo Instituto Brasileiro de Geografia e Estatística (IBGE, 1998) afirmaram ter buscado serviços de saúde em um período de 12 meses, enquanto apenas $46,7 \%$ dos homens o fazem; além disso, 
as mulheres também têm maior número de consultas no mesmo período - principalmente para as adultas fora da faixa etária que designa os idosos - e, mesmo excluindo partos e pré-natal, a realização de exames rotineiros e de prevenção é de $40,3 \%$ das mulheres versus $28,4 \%$ dos homens. Esses dados podem denotar desde desconhecimento e falta de acesso ao sistema até preconceitos e medos arraigados, o que pode ser mitigado por difusão de informação, por exemplo (PINHEIRO RJ, et al. 2002).

\section{Prostatectomia radical}

A prostatectomia radical é um procedimento cirúrgico no qual a próstata é removida totalmente, assim como os tecidos a ela adjacentes e as vesículas seminais, e está indicado quando o tumor se encontra somente na glândula prostática, não atingindo outros órgãos. No momento da indicação cirúrgica, o urologista apresenta os riscos ao paciente, que é orientado a assinar um termo de responsabilidade, ficando assim ciente das complicações futuras (AMERICAN CANCER SOCIETY, 2019).

O documento da Sociedade Brasileira de Urologia (SBU, 2018) explica brevemente em que consiste a cirurgia, com explicação dos termos técnicos - como "remoção de toda a próstata e das vesículas seminais" e "remoção dos linfonodos pélvicos regionais" -; além disso, lista os possíveis riscos associados ao procedimento - impotência sexual, incontinência urinária, dano à parede do reto, embolia pulmonar, infecções, por exemplo - e deixa claro ao paciente que não há garantia de cura do CA.

A assistência subjetiva, respeitando os limites psíquicos e funcionais, contribui para a recuperação gradual ou, até mesmo, adaptações efetivas, colaborando assim para melhor qualidade de vida e reintegração social (SOARES MI, et al, 2015). A condição clínica e o procedimento cirúrgico podem acarretar algumas complicações temporárias e/ou permanentes para o paciente e seu convívio social e familiar. Nessa perspectiva, cabe ao enfermeiro orientar o paciente em relação às etapas do ato cirúrgico (SANTOS J, et al., 2011).

Dentre as cirurgias possíveis para câncer de próstata, existem: as prostatectomias radicais retro púbica e a perineal, sendo a primeira mais invasiva, porém mais efetiva, pois permite a retirada de linfonodos e não costuma gerar problemas de ereção; e as prostatectomias radicais por laparoscopia, em que ocorrem menos incisões e a recuperação é mais rápida, porém também demanda cateter urinário como as cirurgias abertas (AMERICAN CANCER SOCIETY, 2019). Cabe à equipe médica bem informar o paciente, e, estando mais próximo deste, o enfermeiro pode acolher as dúvidas a fim de saná-las, bem como dar apoio ao doente e à família.

No pré-operatório, é importante introduzir medidas educacionais, como suspensão do cigarro em caso de tabagismo, orientar quanto à suspensão de medicações de uso contínuo, como anticoagulante e antiagregante plaquetário, e optar por uma dieta leve (MALUF FC, et al, 2014). Além disso, é preciso garantir que o paciente estará acompanhado durante o transoperatório, reduzindo o medo e a ansiedade e contribuindo para sua estabilidade clínica e seu bem-estar. Tais orientações, mostrando-se efetivas, contribuirão para a recuperação do paciente, inserindo-o em seu próprio cuidado durante o pós-operatório (SANTOS J, et al., 2011).

As sequelas mais comuns ao paciente prostatectomizado são: a incontinência urinária, uma vez que ocorre a lesão ou remoção de fibras musculares, aderidas à próstata que são responsáveis pelo esfíncter urinário externo, uma válvula que impede o escape de urina; e a impotência sexual, que vai variar conforme o grau de comprometimento das artérias localizadas na região e responsáveis pela ereção ou dos nervos responsáveis pela condução de impulsos até o órgão sexual masculino.

Outras sequelas menos comuns, mas que também podem ocorrer, são o estreitamento da bexiga junto à uretra, a embolia pulmonar e a infecção do trato urinário (ITU) (MALUF FC, et al, 2014). Além disso, o paciente pode apresentar distúrbios emocionais, tais como, o medo, a angústia e a incerteza. O enfermeiro tem um importante papel nesse momento, oferecendo aporte emocional ao paciente e à família, a fim de contribuir para o enfrentamento da doença (SANTOS DRF, et al., 2012).

\section{Sistematização da Assistência de Enfermagem}

A Sistematização da Assistência de Enfermagem (SAE) é elaborada pelo enfermeiro individualmente para cada paciente de maneira humanizada e, então, é direcionada aos técnicos e auxiliares que desempenharão suas funções a partir da prescrição do enfermeiro (BRASIL, 1986). 
Existem vários instrumentos metodológicos utilizados para sistematizar a assistência de enfermagem, entre eles, nos planos de cuidados, estão os protocolos operacionais padrão (POP) e o processo de enfermagem (PE), por meio dos quais é possível identificar e estabelecer condutas clínicas convenientes, além de contribuir para a comunicação e a interação entre a equipe multiprofissional, criando um elo em torno às dimensões assistenciais e às gerenciais (SOARES MI, et al, 2015).

De acordo com o Artigo $2^{\circ}$ da Resolução COFEN n 358/2009 (COFEN, 2009), o PE composto por cinco etapas inter-relacionadas, interdependentes e recorrentes. A primeira delas é a etapa de coleta de dados de Enfermagem, que tem o objetivo de conseguir todas as informações do processo saúde doença do paciente e qualquer outra informação importante que a família relate ao enfermeiro.

O Diagnóstico de Enfermagem (DE) é o segundo passo do processo e tem por finalidade agrupar as informações coletadas na primeira etapa e elaborar os cuidados e as intervenções visando ao resultado desejado de maneira acelerada. O enfermeiro consegue tal classificação por meio da Taxonomia de diagnósticos da NANDA Internacional (NANDA-I Heardman Te Kamtisuru, 2018).

A terceira etapa se dá pelo Planejamento de Enfermagem, que determina a elaboração das ações e os cuidados a serem prestados, além dos resultados esperados a partir dessas intervenções. Tais resultados podem ser encontrados nos sistemas de classificação de linguagem que são mais conhecidos e utilizados na realidade brasileira: as taxonomias na Classificação dos Resultados Esperados de Enfermagem (NOC) e as intervenções na Classificação de Intervenções de Enfermagem (NIC).

Importante ressaltar que a taxonomia NANDA, o NIC e NOC são ferramentas internacionalmente padronizadas que têm como objetivo uniformizar a Sistematização da Assistência de Enfermagem (JOHNSON M, et al., 2013).

A implementação caracteriza a quarta fase, na qual são realizados os cuidados planejados, pelo técnico e pelo auxiliar de enfermagem após a avaliação de enfermagem, última etapa do processo, que aponta se as ações e intervenções de enfermagem alcançaram o resultado esperado.

O COFEN ainda ressalva o papel do enfermeiro como líder na implementação do Processo de Enfermagem. Visto que, através de sua observação e orientação é que se obtêm a participação dos demais profissionais de enfermagem na execução do PE (COFEN, 2009).

A aplicação por meio do PE é importante, pois atua de forma hierárquica, a partir do grau de necessidades prioritárias, e pode ser reajustada conforme a exigência assistencial, atendendo assim a todas as necessidades básicas afetadas do paciente, além do processo saúde doença deste.

\section{Intervenções de enfermagem para alta de paciente prostatectomizado}

Foram selecionados alguns diagnósticos de enfermagem, obtidos do NANDA (NANDA-I, 2018), os quais acredita-se serem os que se apresentam com maior frequência em pacientes prostatectomizados, sendo: conhecimento deficiente, disfunção sexual, risco de recuperação cirúrgica retardada, risco de infecção, risco de incontinência urinária de urgência, medo e ansiedade.

As Intervenções de Enfermagem propostas para os diagnósticos identificados foram baseados na tríade NANDA-NOC-NIC (JOHNSON M, et al., 2013) levando em consideração as queixas e complicações dos pacientes. A partir do diagnóstico de enfermagem conhecimento deficiente caberá ao enfermeiro como intervenção aconselhar o paciente melhorando sua disposição em aprender. Além de orienta-lo quanto ao procedimento e tratamento a ser submetido. Como resultado espera-se que o paciente obtenha modificação do comportamento e conhecimento suficiente. (JOHNSON M, et al, 2013).

Para disfunção sexual recomenda-se realizar aconselhamento sexual por meio de esclarecimento de valores; promover apoio emocional para melhora da autoestima e intensificar a melhora da imagem corporal por meio de automodificação. Como resultado, espera-se obter alcance da compreensão transmitida sobre disfunção sexual pós-cirurgia e reduzir comportamentos emocionais decorrentes da cirurgia. (JOHNSON M, et al, 2013)

A intervenção para o risco de recuperação cirúrgica retardada está relacionada à reação emocional pósoperatória, no qual, consiste ao enfermeiro oferecer orientação antecipada sobre os cuidados pós-operatórios, 
desenvolver um plano de alta envolvendo fatores emocionais, além de estimular a participação em grupo de apoio. O objetivo é obter execução efetiva das orientações referidas, manter o paciente esclarecido quanto a fatores que influenciam sua recuperação e melhorar o estado emocional, reduzindo então o risco de recuperação cirúrgica retardada. (JOHNSON M, et al, 2013).

Para risco de infecção, cabe a enfermagem ainda em ambiente hospitalar, avaliar sinais flogísticos de infecção, orientar toda a equipe quanto à importância da lavagem das mãos ao manusear o cateter urinário e os principais cuidados com o mesmo. Diante destas condutas será possível obter como resultado esperado a redução do risco de infecção relacionado ao procedimento invasivo. (JOHNSON M, et al, 2013).

De acordo com o NANDA, 2018-2020, outro diagnostico seria risco de incontinência urinária de urgência relacionada ao regime de tratamento. Como conduta de enfermagem, caberá a equipe, a família ou cuidador estimular a realização de exercícios para musculatura pélvica, promover a criação de hábito urinário e realizar controle hídrico. O resultado esperado seria a melhora funcional da musculatura pélvica e redução de episódios de incontinência urinária e ingesta adequada de líquidos. (JOHNSON M, et al, 2013).

No que concerne a ansiedade que está relacionada à crise situacional, toda a equipe terá como conduta reduzir a ansiedade por meio de técnicas para acalmar e promover apoio à tomada de decisão, com o intuito de manter o autocontrole do paciente e eliminar sensações de apreensão, tensão ou desconforto. (JOHNSON M, et al, 2013; SILVA DM, et al, 2018).

A equipe de saúde deve orientar os pacientes prostatectomizados sobre seu problema, desde o diagnóstico médico até os cuidados domiciliares após a cirurgia. Como orientador, cabe ao enfermeiro encontrar meios que auxiliem a comunicação entre paciente-cuidador-família, garantindo os cuidados que serão prestados em domicílio e contribuindo para a eficácia do tratamento e da reabilitação do doente (POMPEO DA, et al., 2007).

É importante ressaltar que o profissional deverá avaliar o paciente e assegurar-lhe cuidado individualizado, de acordo com suas necessidades e seu estado saúde doença (SILVA DM, et al, 2018). Além disso, terá de prestar cuidados com competência técnico-científica, evidenciada pelos achados clínicos, pela avaliação dos riscos, visando à assistência segura na alta dos pacientes prostatectomizados (SOUZA TM, 2012).

\section{CONSIDERAÇÕES FINAIS}

O presente estudo apresentou de forma simples, o conceito de câncer de próstata, sinais e sintomas da patologia, tipos de tratamento, descrição da prostatectomia e a importância da utilização do processo de enfermagem, mais especificamente um plano de cuidados, que envolve alguns principais diagnósticos de enfermagem, com intervenções e resultados esperados com vistas ao cuidado dos pacientes prostatectomizados na alta hospitalar, e à adequada continuidade dos cuidados em domicílio. Espera-se que este estudo contribua para o embasamento científico na prática dos profissionais de enfermagem que cuidam de pacientes prostatectomizados e estimule-os a utilizar cotidianamente o PE na sua prática.

\section{REFERÊNCIAS}

1. ALMEIDA MDA, et al. Tempo despendido na execução do processo de enfermagem em um centro de tratamento intensivo. Escola Anna Nery, 2012; 16(2): 292-296.

2. AMERICAN CANCER SOCIETY. Following PSA levels during and after prostate cancer treatment, (2019). Disponível em: <https://www.cancer.org/cancer/prostate-cancer/treating/psa-levels-after-treatment.html>. Acesso em: 20 set. 2019.

3. AMERICAN CANCER SOCIETY. Initial treatment of prostate cancer, by stage, (2019) Disponível em: <https://www.cancer.org/cancer/prostate-cancer/treating/by-stage.html>. Acesso em: 20 set. 2019.

4. AMERICAN CANCER SOCIETY. Surgery for prostate cancer, by stage, (2019). Disponível em: < https://www.cancer.org/cancer/prostate-cancer/treating/surgery.html>. Acesso em: 20 set. 2019.

5. BRASIL. Lei $n^{\circ} 7.498$ de 25 de junho de 1986. Dispõe sobre a regulamentação do exercício da Enfermagem e dá outras providências. Diário Oficial da União, Brasília, 26 jun. 1986.

6. CONSELHO FEDERAL DE ENFERMAGEM (BRASIL). Código de ética dos profissionais de Enfermagem: Resolução COFEN n 385/2009. Dispõe sobre a Sistematização da Assistência de Enfermagem e a implementação do Processo de Enfermagem em ambientes, públicos ou privados, em que ocorre o cuidado profissional de Enfermagem, e dá outras providências. Diário Oficial da União, Brasília, 15 out. 2009. 
7. INSTITUTO BRASILEIRO DE GEOGRAFIA E ESTATíSTICA (IBGE). Pesquisa Nacional por Amostra de Domicílios, 1998.

8. INSTITUTO NACIONAL DO CÂNCER. Câncer de próstata, 2019. Disponível em: <https://www.inca.gov.br/tipos-decancer/cancer-de-prostata>. Acesso em: 04 jun. 2019.

9. JOHNSON M, et al. Ligações NANDA NOC-NIC - Condições clínicas: Suporte ao raciocínio e assistência de qualidade. 3. ed. Rio de Janeiro: Elsevier, 2013.

10. MATA LRF, NAPOLEÃO AA. Intervenções de enfermagem para alta de paciente prostatectomizado: revisão integrativa. Acta paul. enferm., 2012; 23(4): 574-579.

11. MALUF FC, et al. Vencer o câncer. São Paulo: Dendrix Sanofi, 2014.

12. MINISTÉRIO DA SAÚDE. Câncer de próstata: causas, sintomas, tratamentos, diagnóstico e prevenção. Saúde de $A$ a Z, 2018. Disponível em: <http://portalms.saude.gov.br/saude-de-a-z/cancer-de-prostata>. Acesso em: 15 maio 2019.

13. MOORE KL, DALLEY AF. Anatomia Orientada para a Clínica. 4 ed. Rio de Janeiro: Guanabara Koogan, 2001 . p.1023.

14. NANDA INTERNATIONAL. Diagnósticos de Enfermagem da NANDA-I: Definições e classificações 2018-2020. 11. ed. Porto Alegre: Artmed, 2018

15. PINHEIRO RJ, et al. Gênero, morbidade, acesso e utilização de serviços de saúde no Brasil. Ciência \& Saúde Coletiva, 2002; 7(4): 687-707.

16. POMPEO DA, et al. Atuação do enfermeiro na alta hospitalar: reflexões a partir dos relatos de pacientes. Acta Paul. Enferm., 2007; 20(3): 345-50

17. ROTHER ET. Revisão sistêmica x revisão narrativa. Acta paul. enferm, jun. 2007; 20(2): v-vi.

18. SANTOS DRF, et al. Cuidados de enfermagem ao paciente em pós-operatório de prostatectomia: revisão integrativa. Rev. Eletr. Enf. [Internet], jul./sep.2012; 14(3): 690-701.

19. SANTOS J, et al. O impacto da orientação pré-operatória na recuperação do paciente cirúrgico. Enfermagem em foco [S.I.], ago. 2011; 2(3): 184-187.

20. SILVA DM, et al. A sistematização da assistência de enfermagem em pacientes com Síndrome de Guillain-Barré. Revista Eletrônica Acervo Saúde, 2018; 1(11): 1151-1157.

21. SMELTZER SC, BARE BG. Brunner \& Suddarth: tratado de enfermagem médico cirúrgica. 12. ed. Rio de Janeiro (RJ): Guanabara Koogan; 2011.

22. SOARES MI, et al. Sistematização da assistência de enfermagem: facilidades e desafios do enfermeiro na gerência da assistência. Escola Anna Nery, mar. 2015; 19(1): 47-53.

23. SOCIEDADE BRASILEIRA DE UROLOGIA (SBU). Consentimento Informado da SBU, 2018. Disponível em: <http://portaldaurologia.org.br/medicos/meu-consultorio/consentimentos-informados/>. Acesso em: 20 set. 2019.

24. SOUZA TM, et al. Diagnósticos, prognósticos, e intervenções de Enfermagem na sala de recuperação pós-anestésica. Rev. SOBECC, São Paulo. out./dez 2012; 17(4): 33-47 